\title{
Argumentación y toma de decisiones en Trabajo Social: Desafíos para la formación profesional
}

\section{Argumentation and decision making in Social Work: Challenges for Social Work education}

\begin{abstract}
Fabiola Cortez-Monroy / Leonardo Oneto / Isabel SaAvedra / María Olga Solar
Fabiola Cortez-Monroy, PhD @ e en Sociología, es académica de la Escuela de Trabajo Social de la Universidad Católica de Chile, su dirección postal es Universidad Católica, Campus San Joaquín Av. Vicuña Mackenna 4860; su dirección electrónica es: fcortezm@uc.cl

Leonardo Onetto es Asistente Social, Doctor en Lingüística. Departamento de Lingüística y Ciencias del Lenguaje. Universidad Católica de Valparaíso. Actualmente es profesor de la Escuela de Trabajo Social, Pontificia Universidad Católica de Valparaíso. Isabel Saavedra es Asistente Social, Master in Education for Social Work, de The National Catholic School of Social Service de The Catholic University of America. Washington. Actualmente es profesora de la Escuela de Trabajo Social Pontificia Universidad Católica de Valparaíso.

María Olga Solar es Asistente Social, Master in Education for Social Work, de The National Catholic School of Social Service de The Catholic University of America. Washington. Actualmente es Profesora de la Escuela de Trabajo Social de la Pontificia Universidad Católica de Chile.
\end{abstract}

\begin{abstract}
Resumen
Este artículo discute, a partir de los hallazgos previos de la investigación "Infancia y riesgo social. Argumentos que utilizan los trabajadores sociales para tomar decisiones frente a situaciones de riesgo social que afectan al niño en su familia"1, la necesidad de repensar la formación de los estudiantes de Trabajo Social introduciendo marcos conceptuales que les permitan comprender y mejorar los procesos a través de los cuales los profesionales toman decisiones en aquellas situaciones en las que les corresponde intervenir. Dos enfoques conceptuales son relevados: la teoría de la argumentación y la práctica basada en la evidencia.

Palabras claves. argumentación, toma de decisiones, formación en Trabajo Social, práctica basada en la evidencia.

Abstract

This article is based on previous findings of the investigation "Children and social risk, arguments used by social workers to make decisions in situations of social risk that affect children and their families", and discuss the need to rethink the training of students entering social work, introducing conceptual frameworks that enable them to understand and improve the processes through professionals make decisions. Two conceptual approaches were surveyed: the theory of argumentation and evidence-based practice.
\end{abstract}

Key words. argumentation, decision making, social work education, evidence based practice.

\section{Introducción}

Este artículo trabaja sobre los resultados preliminares de la investigación "Infancia y riesgo social. Argumentos que utilizan los trabajadores sociales para tomar decisiones frente a situaciones de riesgo social que afectan al niño en su familia”. Su propó- sito es analizar la importancia de la argumentación y toma de decisiones en Trabajo Social, reflexionando en torno a la necesidad de repensar la formación profesional.

En la investigación citada, se analizan y comparan los argumentos explícitos que ofrecen los trabaja-

1 Investigación presentada en el 33 Congreso Mundial de Escuelas de Trabajo Social, realizado en Santiago, Agosto de 2006. 
dores sociales cuando ponderan y toman decisiones respecto a la forma de enfrentar situaciones de riesgo social que afectan a los niños en sus familias. Cabe señalar, que la investigación replica en Chile una línea iniciada por Rujla Osmo en Israel y proseguida en Canadá, la cual busca comprender y mejorar los procesos a través de los cuales los profesionales toman decisiones en aquellas situaciones en las que les corresponde intervenir. No se trata de evaluar si las decisiones que los profesionales adoptan, son o no correctas, sino de profundizar en las razones que ellos ofrecen para ponderar una situación y proponer una intervención. La comparación se realizó contrastando los resultados obtenidos en Chile con aquellos logrados en las mismas dimensiones por Rujla Osmo en su investigación "Children at risk: Rationales for risk assessments and interventions" ${ }^{\text {. }}$. Para analizar los argumentos explícitos que ofrecen los trabajadores sociales, se utilizaron los modelos propuestos por Stephen Toulmin $(1984,2007)$ y Aaron Rosen et al. (1995), los cuales permiten examinar tanto los contenidos como la estructura de sus argumentos.

Se asumió que por tratarse de una investigación pionera en Chile, sus resultados debían ser considerados como iniciales y exploratorios. Sin embargo, ellos permitieron abrir la discusión sobre temas importantes acerca de la formación en Trabajo Social, como lo es la incorporación de enfoques que permitan fortalecer los procesos de argumentación y de toma de decisiones en la intervención social.

El artículo ha sido estructurado en tres partes. En la primera de ellas se analiza conceptualmente la racionalidad de la argumentación. En la segunda, se presentan algunos de los principales hallazgos de la investigación, analizando la argumentación y toma de decisiones en Trabajo Social. Finalmente, se aborda la formación profesional y el desafío de mejorar los procesos de argumentación y toma de decisiones. Al respecto, se apela a la teoría de la argumentación y a la práctica basada en la evidencia.

\section{Racionalidad de la argumentación}

La argumentación está presente en la vida cotidiana, pues permanentemente la persona se enfrenta la necesidad de justificar, fundar o dar credibilidad a sus afirmaciones, de modo que éstas sean consideradas en su entorno social o profesional. "La argumentación implica razonamiento. Aristóteles fue uno de los primeros en descubrir la existencia de una lógica argumentativa, de naturaleza inductiva en los discursos sociales, diferente a la silogística y valorada en la actualidad en función de parámetros como coherencia y adecuación. Una línea de argumentación inductiva permite inferir a partir de una evidencia particular con el fin de derivar unas conclusiones" (Rodríguez, 2004: 3).

Por argumentación, se entiende la actividad de realizar aserciones, cambiándolas, apoyándolas con razones, criticando estas razones y refutando las críticas (Toulmin et al., 1984). Involucrarse en las acciones de justificar los propios puntos de vista y enfrentar la oposición, introduce en el campo psicológico del individuo un nuevo objetivo de reflexión, a saber, sus propias cogniciones respecto al fenómeno del mundo (Leitão 2007). La argumentación "contempla la exposición de una tesis controvertida, el examen de sus consecuencias, el intercambio de pruebas y de buenas razones que la sostienen y una clausura bien o mal establecida" (Marafioti, 1998: 216).

El argumento es una aserción/declarativa, en la compañía de su justificación (Osmo \& Benbennishty, 2002). Toulmin (2007), define seis tipos de declaraciones. Las tres primeras constituyen el nivel básico del argumento:

- Conclusión: declaración de una afirmación o aserción

- Dato: provee a la conclusión de evidencias

- Ley de pasaje: garantía o justificación de la conexión entre el dato y la conclusión por medio de la apelación a una ley de inferencias

Las otros tres tipos de declaraciones, conforman el argumento complementario:

- Modalizador: expresa los grados de confianza y probabilidad de que la conclusión sea cierta.

- Refutación: señala bajo qué condiciones no se sostiene la conclusión. Introduce reservas mostrando los límites a la aserción.

- Soporte: justificaciones, respaldos o apoyo a la garantía, mediante la apelación a generalizaciones, que explicitan el cuerpo de conocimientos usados para establecer la confiabilidad de la garantía Ej.: datos empíricos, conocimiento común, práctica profesional o teoría científica.

El marco conceptual propuesto por Toulmin (2007) es posible complementarlo -dada la utilidad para nuestra disciplina - con la propuesta de Rosen y sus

2 Hebrew University, Jerusalem Israel, junio 2002. 
colaboradores (1995), quienes analizan el uso del conocimiento realizado por los trabajadores sociales en el proceso de tomar y justificar las decisiones prácticas. Rosen et al. (1995), identifican tipos de conocimientos usados por estos profesionales al justificar sus decisiones prácticas:

- Conocimiento General: declaraciones de conocimientos personales adquiridos articulados en términos generales, sin especificar su origen.

- Teoría: declaración que invoca o menciona con nombre una teoría o un teórico o un vínculo entre dos conceptos profesionales.

- Política: cualquier declaración referente a reglas, normas o directivas del servicio, agencia u otra unidad administrativa.

- Valor: declaración de valor o aserción categorial de verdad o norma universal.

- Evidencia empírica: afirmación de evidencia empírica (publicada o no) o referente a un "estudio".

- Experiencia práctica: mención a la práctica propia y de colegas, o la práctica colectiva de varios colegas.

En relación al conocimiento proveniente de la teoría, Rosen et al. (1995) distinguen tres tipos de conocimientos: descriptivo, explicativo y de control.

El conocimiento descriptivo: guía a los trabajadores sociales para clasificar los fenómenos que encuentran, en categorías conceptuales significativas. El conocimiento descriptivo incluye la información sobre las características, los indicadores o las incidencias del fenómeno en la preocupación pro- fesional (por ejemplo, pobreza, maltrato infantil, comportamiento mal adaptativos, los desórdenes mentales, violencia en la comunidad, entre otros).

El conocimiento explicativo: es aquel que proporciona la profundidad para entender los fenómenos a tratar, sus dinámicas, los factores que influencian su variabilidad, y sus consecuencias. Alerta a los trabajadores sociales acerca de los factores que contribuyen probablemente al desarrollo y a la persistencia de problemas y, lo más importante, provee a los profesionales de una base para predecir el tipo y el grado de otras condiciones indeseables, asociadas probablemente a problemas. La comprensión de esa dinámica guía las decisiones de los trabajadores sociales acerca de si la intervención es necesaria y en qué condiciones resulta indicada.

Cabe señalar que el conocimiento de tipo descriptivo y explicativo se requiere para evaluar, explicar o anticipar el curso natural de los acontecimientos (incluyendo los vistos como problemas), esto es proporcionando explicaciones ex post facto con respecto a factores o antecedentes, o prediciendo las consecuencias naturales de los acontecimientos.

El conocimiento de control: permite a los trabajadores sociales controlar un fenómeno de preocupación, esto es, la capacidad de mantener su curso deseado. El conocimiento satisface sus funciones de control, cuando es capaz de guiar a los profesionales en la selección y puesta en práctica de las intervenciones que logran con éxito los resultados deseados.

El modelo de análisis, considerando el marco propuesto por Stephen Toulmin $(1984,2007)$ y Aaron Rosen et al. (1995), queda como se presenta a continuación:

ANÁLISIS DE LA ARGUMENTACIÓN

(Modelo de Stephen Toulmin - Categorías de Aaron Rosen et al.)

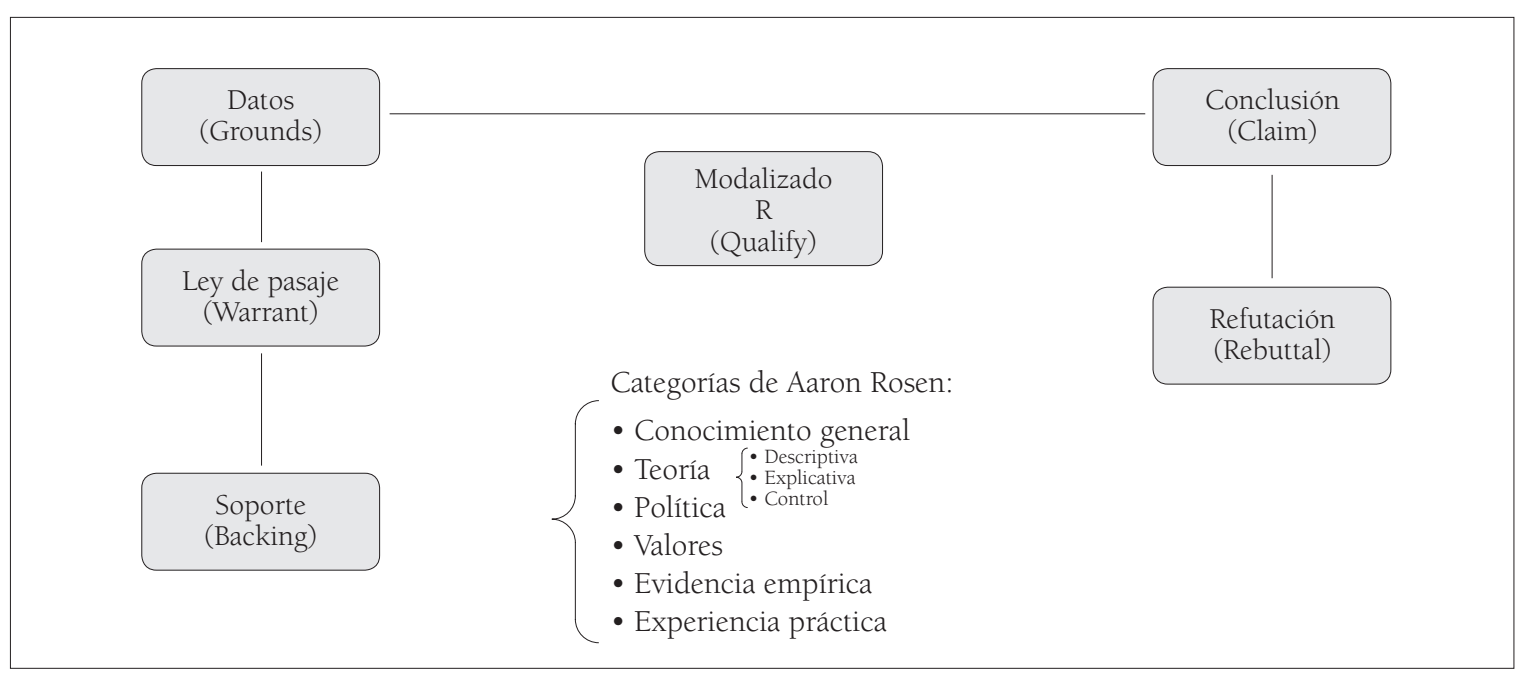


El modelo de Toulmin $(1984,2007)$, permite examinar tanto los contenidos como la estructura de los argumentos, identificando si ellos son de nivel básico o complementario. Esta estructura de análisis, devela el nivel de complejidad de la argumentación. Una argumentación compleja es aquella que es capaz de precisar mejor las condiciones bajo las cuales un evento ocurre y la ponderación de la probabilidad de su ocurrencia. De esta forma, ella refleja mayor dominio del campo decisional, permitiendo inferir con mayor seguridad que las decisiones están fundadas en un juicio profesional adecuado. Asimismo, conocer el tipo de justificación o fundamento al que se apela cuando se establece un juicio diagnóstico o decide una intervención específica denotaría un mayor o menor dominio de conocimientos del tipo predictivo validado, en este caso se trata de la calidad del argumento, es decir, de la validación disciplinaria de éste (Osmo \& Benbennishty, 2002).

\section{Argumentación y toma de decisiones en Trabajo Social}

En la investigación "Infancia y riesgo social. Argumentos que utilizan los trabajadores sociales para tomar decisiones frente a situaciones de riesgo social que afectan al niño en su familia", se analizaron y compararon los argumentos explícitos que ofrecen los trabajadores sociales cuando ponderan y toman decisiones respecto a la forma de enfrentar situaciones de riesgo social que afectan a los niños en sus familias. La idea no era evaluar si las decisiones que los profesionales adoptan son o no correctas, sino profundizar en las razones que ellos ofrecían para ponderar una situación y proponer una intervención. La comparación se realizó contrastando los resultados obtenidos en Chile con aquellos logrados en las mismas dimensiones por Rujla Osmo en su investigación "Children at risk: Rationales for risk assessments and interventions", realizada en el año 2002 en Israel.

La muestra de la investigación contempló a 52 Trabajadores Sociales de las regiones Quinta y Metropolitana, que trabajaban con niños vulnerados en sus derechos y cuya función principal era diagnosticar su situación. La experiencia profesional promedio del grupo es de 8,5 años y la experiencia promedio en el ámbito de la infancia, igual a 6,5 años. La recolección de la información se realizó entre los meses de enero y mayo de 2006.

Cabe señalar que en la intervención con la infancia en riesgo social, el diagnóstico es considerado un momento clave. Él constituye una de las puertas de ingreso de la demanda de atención de los niños/as y sus familias (SENAME, 2005). El Servicio Nacional de Menores de Chile, define el diagnóstico como "una acción centrada en el niño/a y adolescente víctima de vulneración de sus derechos y sus familias, y que está enmarcada en la protección integral y la Convención de los Derechos del Niño. Es un tipo de atención que se debe realizar en el más breve plazo y preferentemente en forma ambulatoria, de manera que los niños/as no sean desarraigados de sus familias y localidades de pertenencia" (SENAME, 2005).

Para analizar la racionalidad en el proceso de toma de decisiones de los trabajadores sociales, se asumió un enfoque de "incidente crítico". Se trata de una estrategia común para estudiar el proceso de toma de decisiones (Drury-Hudson, 1999; Osmo $\&$ Rosen, 2002, en Osmo \& Benbennishty, 2002). Para ello se presentó a los profesionales un caso de análisis que muestra la situación de un niño y su familia. Este caso fue el mismo usado por Osmo, R. Benbennishty R, (2002) en su investigación "Children at risk: Rationales for risk assessments and interventions". El contenido del caso se elaboró a partir de registros originales de situaciones auténticas de Israel. En dicho caso se relata una situación ambigua que requiere discreción y una deliberación cuidadosa. Como una forma de asegurar la autenticidad del caso, se realizaron pequeños ajustes de modo de adaptarlo a la realidad chilena.

En la investigación en Chile, siguiendo a Osmo, R. $\&$ Benbennishty R, (2002), quienes a su vez continúan la línea de investigación de Berbenishty \& Shapira (1993, en Osmo \& Benbennishty, 2002), se estudió la racionalidad proveniente de dos tipos de decisiones: el grado de riesgo en el niño y la recomendación de la intervención. El grado de riesgo en el niño, fue medido en una escala de Likert, en un rango de 1 (sin ningún riesgo) a 6 (riesgo extremo).

De esta forma, para analizar la racionalidad dada por los profesionales, se utilizaron dos aserciones: la evaluación del riesgo y la intervención recomendada. El análisis de la estructura de la racionalización, se llevó a cabo revisando las razones que los profesionales ofrecían su evaluación de riesgo e intervención recomendada. Se indagó en los seis componentes del argumento, tomando como base la conceptualización de Toulmin: Aserción (“¿Qué pide usted?"), Evidencias ("iQué dato tiene?"), Garantía (iQué garantiza su conclusión?"), Soporte ("Cual es la justificación de su línea argumentativa?"), Refutación ("¿Bajo que condiciones su argumento se desmoro- 
na, haciendo que usted debiera cambiar su idea?"). El análisis del contenido del componente soporte, se realizó considerando como referente conceptual Rosen et al. (1995). Se consideraron las seis categorías propuestas por este autor y el número de veces en que cada profesional las utilizó: conocimiento general, teoría (descriptiva, explicativa o de control), política, valor, evidencia empírica y experiencia práctica.

Cabe señalar que dado que esta investigación es pionera en Chile en analizar el contenido de los argumentos y las razones de los Trabajadores Sociales para tomar decisiones en el campo de los niños en riesgo social, sus resultados, deben ser considerados como iniciales y exploratorios. Hecha esta salvedad, se ofrecerá a continuación una síntesis de la discusión de los principales resultados.

$\mathrm{Al}$ analizar los componentes utilizados por los Trabajadores Sociales, se observó que prácticamente la totalidad de los profesionales formulan argumentos de nivel básico. Esto se ve reflejado en que el 100\% de los profesionales presentan evidencias del caso, consideradas relevantes para avalar sus juicios, y la mayoría se refiere a alguna regla de inferencia que explicaría la conexión entre la evidencia que ellos ofrecen y el juicio que realizan, es decir, presentan ley de pasaje. Esto muestra que los profesionales al momento de emitir un juicio, consideran las evidencias del caso y las articulan de acuerdo a ciertas reglas que estiman relevantes. Tal como lo expresan Osmo \& Benbennishty (2002), sus respuestas no son automáticas o solamente burocráticas; ellas están basadas en el examen de las características específicas del caso.

En el nivel complementario de argumentación, el cual contempla soporte, refutación y modalizador, se observa una situación muy diferente, constatándose que la mayoría de los trabajadores sociales no logra este nivel. En efecto, sólo un 8\% de los trabajadores sociales de Chile y un 6\% de los profesionales de Israel, cuando ponderan el riesgo del niño, alcanzan este nivel argumentación. Al momento de proponer una intervención, el panorama anterior se reproduce para el caso de Israel, donde están más ausentes la refutación y los modalizadores, alcanzando este nivel solo un 13\% de los profesionales. Sin embargo, en el caso de Chile este porcentaje aumenta, llegando a un 22\%, debiéndose ello básicamente a que el componente refutación adquiere mayor presencia en este grupo.

Es preciso llamar la atención sobre el bajo porcentaje de profesionales que logra el nivel complementario de argumentación, pues como lo expresa Osmo \&
Benbennishty (2002), dicho nivel refleja competencias basadas en la educación y el entrenamiento, que son claves para la argumentación profesional.

Especialmente ausente estuvieron la refutación y los modalizadores. Para el caso de Chile, esto es más evidente al momento en que los profesionales evalúan el riesgo. Estos componentes adquieren mayor presencia, cuando los profesionales son estimulados a entregarlos. Los resultados parecen sugerir que los trabajadores sociales no estarían usando ese razonamiento complejo que tendemos a atribuirle (e.g.Drury \& Hudson,1999; Shanteau, 1992 en Osmo \& Landau, 2001). En efecto, estos componentes mejoran la probabilidad de que se emitan juicios profesionales más sólidos. Cabe señalar, que los modalizadores, remiten a grados de confianza en los juicios, reconociendo la posibilidad de un error. Por su parte, las refutaciones consideran posibles escenarios alternativos, y posibles cambios en el contexto, que llevaría a requerir de ajustes y modificaciones en el juicio Osmo \& Benbennishty (2002).

El bajo nivel de refutaciones presentes en las argumentaciones de los trabajadores sociales, cuando ellas no son inducidas, podría evidenciar, como lo plantea Osmo \& Landau (2001), que los trabajadores sociales son vulnerables a confirmar prejuicios, que podrían impactar negativamente en la toma de decisiones. Esto último reforzaría la tesis de esta investigación, en el sentido "a igual nivel de complejidad en las situaciones a intervenir, un elemento diferenciador en la evaluación del riesgo social y en los cursos de acción propuestos, es el tipo de respaldo y estructura argumental utilizada por el trabajador social". Cabe señalar, que el marco conceptual propuesto por Toulmin, supone aceptar hacer de la verdad un concepto no binario (verdadero/falso), sino gradual (Marafioti, 1998: 215). Al incluir la refutación en el proceso de toma de decisiones, el Trabajador Social permanece atento y abierto a considerar cómo información nueva y diferente podría impactar en la decisión (Osmo \& Benbennishty, 2002).

El uso mínimo de refutaciones debiera interpelar a la formación profesional de los trabajadores sociales actualmente en terreno y a la educación del Trabajo Social, de modo de frente a este hecho y fortalecer el entrenamiento que se centra en las habilidades de pensamiento crítico. El pensamiento crítico se define y caracteriza por ser una destreza de tipo cognitiva que cuestiona, pone en tela de juicio y problematiza cualquier verdad o conocimiento que, sin un juicio crítico previo, contextualizado, 
pretenda erigirse como único, definitivo y absoluto (Miranda, 2003).

Por otra parte, como se indicó anteriormente, esta investigación indagó en el contenido de los argumentos que realizan los trabajadores sociales. Para ello, se utilizó como referente conceptual el marco propuesto por Rosen et al (1995), que permite identificar el tipo de conocimiento que fundamenta sus razonamientos (juicios y toma de decisiones) en casos de riesgo social.

A igual que en el caso de Israel, en Chile la categoría más usada como soporte de la evaluación del riesgo es el "conocimiento general". En la intervención recomendada, los soportes se diversifican. De esta forma, si bien la mayoría usa el conocimiento general, también adquieren fuerza como soporte la experiencia profesional y la política.

Ahora bien, a diferencia de los hallazgos encontrados en Israel, en que existe una tendencia por parte los trabajadores sociales a equilibrar en sus juicios (Ver Tabla $N^{\circ} 1$ ), el uso del conocimiento general con el conocimiento orientado a la teoría, para el caso de Chile, solo una profesional usa explícitamente la teoría como soporte de su argumentación (Ver tabla $\mathrm{N}^{\circ} 1$ ). Al analizar la teoría utilizada por el profesional, según tipos (descriptiva, explicativa y de control) propuestos por Rosen et al. (1999), se aprecia que ella es descriptiva, en el sentido que es utilizada como una guía para clasificar los fenómenos en categorías conceptuales significativas. Este tipo de teoría incluye la información sobre las características, los indicadores, o las incidencias del fenómeno en la preocupación profesional (por ejemplo, pobreza, abuso de niños, los desórdenes mentales, violencia en la comunidad, entre otros).

Este resultado, invita a continuar la exploración en esta línea, pues para el contexto chileno existe sólo un estudio con el cual se podrían contrastar los resultados de esta investigación. Dicho estudio (Miranda, 2006), aborda el rol de las teorías éticas en los procesos decisionales. En él se examinan los argumentos de 103 trabajadores sociales de Chile, relativos a sus opciones, en términos de principios éticos en general y dilemas éticos en particular. El autor muestra que la argumentación dominante observada en los discursos de los trabajadores sociales que constituyeron la muestra, es "no teórica". Al analizar sus argumentos, Miranda (2006) constata que solo un $32 \%$ de ellos tienen una relación de adecuación con las teorías éticas estudiadas.

Por otra parte, no deja de llamar la atención que tanto los profesionales de Israel como los de Chile, no utilizan la evidencia empírica como soporte de sus juicios profesionales, es decir, no avalan sus argumentaciones apelando a la investigación empírica. Una posible explicación de estos resultados es que los trabajadores sociales si usan el conocimiento empírico, pero no están habituados a citar estos resultados en un discurso coloquial Osmo \& Landau, 2001). Otros autores observan que el uso de los resultados de la investigación es un proceso mucho más variado, complejo, sutil e indirecto que lo pensado anteriormente, y no se captura solitariamente por referencia directa sobre

TABLA N ${ }^{\circ} 1$

CATEGORÍAS DE CONOCIMIENTO UTILIZADAS POR LOS TRABAJADORES SOCIALES COMO SOPORTE EN LA PONDERACIÓN DEL RIESGO Y EN LA INTERVENCIÓN RECOMENDADA (EN \%)

\begin{tabular}{|l|c|c|c|c|}
\hline \multirow{2}{*}{ País } & \multicolumn{2}{|c|}{ Chile } & \multicolumn{2}{c|}{ Israel $\left(^{*}\right)$} \\
\hline Categorías & $\begin{array}{c}\text { Evaluación } \\
\text { del riesgo }\end{array}$ & $\begin{array}{c}\text { Intervención } \\
\text { recomendada }\end{array}$ & $\begin{array}{c}\text { Evaluación } \\
\text { del riesgo }\end{array}$ & $\begin{array}{c}\text { Intervención } \\
\text { recomendada }\end{array}$ \\
\hline Conocimiento general & $\%$ & $\%$ & $\%$ & $\%$ \\
\hline Teoría & 42,3 & 55,8 & 46 & 25 \\
\hline Experiencia & 1,9 & 1,9 & 38 & 29 \\
\hline Valores & 0 & 42,3 & 9 & 22 \\
\hline Evidencia empírica & 0 & 11,5 & 7 & 15 \\
\hline Política & 0 & 0 & 0 & 0 \\
\hline
\end{tabular}

(*) Osmo, R. Benbennishty R., (2002). Children at risk: Rationales for risk assesments and interventions. Hebrew University, Jerusalem Israel. 
un estudio particular (Fortune \& Reid 1999 en Osmo \& Landau, 2001). Así es posible que los hallazgos empíricos sean internalizados y asimilados en el proceso de pensamiento en tal medida que sean considerados conocimiento general. Entonces, cuando los trabajadores sociales dijeron que usaron el conocimiento general, ellos pueden haber estado refiriéndose a la investigación empírica (Osmo \& Landau, 2001). Para Rujla Osmo \& Landau (2001), refiriéndose al caso de Israel, la posibilidad de que los trabajadores sociales no usen evidencia de investigación en la práctica, tal vez sea la más probable explicación. Según la autora, el patrón total de los hallazgos indica que los trabajadores usan una mezcla de conocimiento basado en la práctica y la teoría, y no basan sus juicios en ningún grupo específico de estudios empíricos relevantes.

Otros autores, refiriéndose a ámbitos diferentes, llegan a conclusiones similares. En efecto, Joaquín Brunner (1993), cita un estudio de Guillermo Briones (1990), referido a Chile en el área de la educación. En dicha investigación, que abarca el período 1980-1989, se entrevistaron 41 investigadores educacionales -un 90\% contestó positivamente a la pregunta sobre si "algunos resultados de sus investigaciones han tenido alguna forma de utilización, cualquiera sea su modo o nivel". Sin embargo, un $80 \%$ de ellos estima que en Chile, en general, el nivel de utilización de los resultados de la investigación educacional es "bajo", mientras que los demás sostienen que es apenas "regular". Este mismo estudio se replicó -pero esta vez se trabajó sobre la base de una pequeña muestra de altos decidores del Ministerio de Educación. En él, se arriba a la conclusión de que el uso efectivo de resultados de investigación, en ese ámbito, es en extremo modesto. Los decidores justifican ese bajo nivel de utilización con argumentos que son conocidos:

- Debido a que a la hora de usarlos, los resultados no están disponibles.

- Debido al recargo de acciones de administración de corto plazo y la falta de equipos estables de asesoría que procesen la información.

- Debido a los constantes cambios en la investigación y su tendencia a "autoalimentarse" de investigaciones similares.

- Debido a la ausencia de investigaciones pertinentes.

- Debido a la desconfianza que provocan investigaciones cuyas premisas se expresan como dogmas, etc.
Brunner (1993) después de constatar que los decidores no tenían conocimiento prácticamente de ninguna de las investigaciones publicadas a lo largo del período 1980-1989, concluye con una cita de Briones (1990), señalando que "no hay utilización de los conocimientos generados por esas investigaciones en la formulación de políticas y toma de decisiones a nivel superior".

En lo relativo al uso de los valores como fundamento de la argumentación profesional, se observa que tanto los trabajadores sociales de Chile como Israel, apelan escasamente a este componente, esto se acentúa aún más en la evaluación del riesgo. En la recomendación de la intervención, el uso de los valores adquiere mayor presencia. Osmo (2003), llama la atención acerca de la posibilidad de que los trabajadores no estén concientes de la dimensión del valor de sus opciones. A juicio de la autora es imperativo que los trabajadores sociales estén concientes de los valores que impactan sus juicios, y que sean capaces de articular y presentar a sí mismos y a otros, los valores que sustentan sus juicios. Esto es importante en la mayoría de las áreas de intervención, pero es esencial en el área de la protección de la infancia. Las decisiones en esta área, tales como apartar al niño de su familia biológica, involucran asuntos éticos y de valor, que los profesionales necesitan conocer. Ellos requieren saber cuáles son las fuentes de sus juicios, y no atribuirlos a 'expertise objetiva' (Clark \& Asquito, 1985; Maas, 1994 en Osmo y Landau, 2001). De acuerdo a Miranda (2006), darse cuenta del propio punto de vista moral, aparece como un indicio de la mayor o menor reflexividad de los trabajadores sociales en su quehacer profesional. Profundizar en el análisis de la influencia de los valores personales en el proceso de toma de decisiones es relevante, pues como lo plantea Miranda, "reconocerse en un punto de vista moral, posibilita la articulación crítica entre los valores profesionales y los valores personales" (2006: 96).

\section{Pensar la formación en Trabajo Social}

Los resultados obtenidos en la investigación interpelan los modos de comprender y de formar en Trabajo Social. Para intervenir es preciso ver y comprender qué es esa interpretación, y que sus formas están influyendo fuertemente sobre ese horizonte de intervención (Matus en Ludi, 2003: 20).

Los hallazgos descritos en el punto anterior ponen de manifiesto la relevancia que tiene la teoría de la argumentación para la toma de decisiones en Traba- 
jo Social. Los resultados evidencian la necesidad de formar a los estudiantes de la disciplina en marcos conceptuales que les permitan comprender y mejorar los procesos a través de los cuales los trabajadores sociales toman decisiones en aquellas situaciones en las que les corresponde intervenir. El interés en la argumentación al tomar una decisión, radica en que cuando ella está articulada en detalle, es posible evaluar su justificación, aprender de ella y, tal vez, identificar errores que deben rectificarse (Osmo \& Landau, 2001). El ejercicio de producción de argumentos orales y textuales facilita a los estudiantes el fortalecimiento de sus competencias comunicativas, además de potenciar el "metadiscurso en sus proceso de aprendizaje" (Sardá \& Sanmartí, 2000 en Valle \& Cabrera, 2007:151).

Involucrarse en las acciones de justificar los propios puntos de vista y enfrentar la oposición, estimula al sujeto a reflexionar sobre sus propias cogniciones respecto al fenómeno del mundo. Asimismo, la reflexión sobre los fundamentos y límites de las propias afirmaciones, le permiten tomar conciencia y aumentar el control sobre su propio conocimiento (Leitão, 2007).

Estimular en los estudiantes lograr un nivel complementario de argumentación -en el sentido de Toulmin $(1984,2007)$ - resulta clave para su ejercicio profesional. Ello porque este nivel de argumentación es el más complejo, incluyendo tres componentes: soporte, refutación y modalizador. Son justamente estos componentes, aquellos que favorecen la solidez de los juicios, previniendo la necesidad de introducir ajustes y modificaciones en los mismos. Lograr este nivel de argumentación, implica alcanzar un nivel de pensamiento complejo, reflexivo y crítico. "El aporte fundamental de la teoría de la argumentación racional consiste, precisamente, en renunciar a la postulación de una verdad en sentido fuerte y centrase en las pretensiones de corrección, en tanto susceptibles de crítica y fundamentación por parte de los participantes (Aldao, 2007:14).

Ahora bien, Valle y Cabrera insisten en que "la familiarización de los estudiantes durante un breve período de clases con las técnicas de la argumentación, no logrará desarrollar a cabalidad la habilidad del pensamiento crítico y creativo. Este es un proceso que demanda práctica a través de actividades pedagógicas, sostenibles en el tiempo y en todas las asignaturas, pues argumentar es una competencia transversal a todas las asignaturas" (2007:151).

La propuesta de Toulmin $(1984,2007)$ resulta efectiva en el proceso de enseñanza-aprendizaje, pues al permite comprender el proceso de formar una argumentación, explicitando los elementos de la misma. "Ella se adapta convenientemente a la práctica de la enseñanza en que el estudiante debe reflexionar sobre la estructura del texto argumentativo que construye a partir de los datos o fenómenos observados, justificando con razones (argumentos) y fundamentando con los principios y leyes científicamente aceptados (garantía). Acciones que refuerzan en el estudiante su capacidad para establecer relaciones entre ideas, generar hipótesis y reflexionar sobre las mismas" (Valle y Cabrera, 2007: 151). En el marco del contexto académico y profesional, en los que se preserva, genera y difunde conocimiento, la argumentación lógica es una condición intrínseca del discurso que le aporta solidez y prestigio a su autor (Rodríguez, 2004). De ahí la necesidad entonces de dedicar esfuerzos al ejercicio argumentativo por parte de los estudiantes en su proceso de formación.

Asimismo, los resultados de la investigación evidenciaron una clara tendencia por parte los trabajadores sociales que formaron parte de la muestra a sustentar sus juicios en el conocimiento general, más que en la teoría, no apelando a investigaciones empíricas relevantes para su intervención. Esto desafía a pensar en incorporar a la formación en Trabajo Social, metodologías que favorezcan el uso de la teoría y de la evidencia proveniente de la investigación en el ejercicio profesional.

En los últimos años en los Estados Unidos, Australia, Canadá y el Reino Unido se ha expandido en Trabajo Social una corriente llamada Práctica Basada en la Evidencia (Evidence-Based Practice), que como su nombre lo indica propone sustentar la toma de decisiones en evidencias. Esta tendencia proviene de Medicina Basada en la Evidencia (MBE). Afirmaciones centrales de la MBE son, que existe un cúmulo de evidencias científicas que deben ser la base de la práctica clínica y que las prácticas clínicas y en poblaciones deben someterse al análisis científico (Jenicek M., 1997 en Bedregal P., Cornejo C., 2005: 997).

De esta forma, la evidencia proveniente de la investigación es considerada una elemento central en la toma de decisiones, integrando, además, en este proceso todos los otros elementos necesarios para tomar una decisión (culturales, sociales, valóricos), así como las diversas disciplinas y enfoques que se requieran para que la decisión que se tome sea la más adecuada a las condiciones y necesidades de los pacientes (Biagini, 2005).

Para Couturier y Carrier (2003), en los servicios sociales, la práctica basada en la evidencia, pue- 
de comprenderse como el concienzudo, explícito y juicioso uso de la mejor evidencia actual en la toma de decisiones en relación con el bienestar de las personas que necesitan de los servicios sociales. Cabe señalar que para estos autores una evidencia es el producto indiscutible de una actividad de investigación (Couturier \& Carrier, 2003). Sin embargo, autores como Bedregal y Cornejo, relevan la necesidad de que "el concepto de «evidencia» debe ser separado de su legado positivista lógico, para incluir también lo que es evidente desde el contexto, la comunidad, y desde otras metodologías además de la experimental. La evidencia, así entendida, nos abre la posibilidad de mirar cómo el significado del lenguaje se modifica respecto de la posición del sujeto (Cornejo, 2004). La gran oportunidad que nos otorga la polisemia del concepto, es recrearla desde abajo hacia arriba, explicitar las distintas suposiciones de práctica de investigación y aplicación clínica cotidiana y examinar los vínculos existentes entre los diversos niveles" (Bedregal $\&$ Cornejo, 2005: 981).

Cabe señalar que en Trabajo Social la perspectiva de la práctica basada en la evidencia es incipiente. Así como ella ha sido objeto de elogios, también ha recibido objeciones referidas, entre otros aspectos, a los obstáculos para ser asumida como enfoque. $\mathrm{Al}$ respecto se pueden mencionar algunas de estas dificultades identificadas por Morago (2004):

- La existencia de obstáculos materiales y organizativos para la implementación de este modelo de la en el área del Trabajo Social. Dentro de éstos, se encuentra la presión bajo la cual, frecuentemente, los trabajadores sociales desarrollan su labor.

- La falta de preparación técnica de un amplio número de profesionales a fin de evaluar críticamente los resultados de investigación

- La presencia de factores psico-sociales: temor a cuestionar las opiniones predominantes, en un clima en el que las organizaciones son gestionadas, cada vez más, de acuerdo a criterios gerenciales, no siempre comprensivos con un juicio profesional que cuestione los procedimientos de deliberación y decisión establecidos (Oxman y Flottorp, 2001 en Morago 2004).

- El contexto de complejas -y, a veces, caóticasrelaciones humanas, y en el que, con frecuencia, condiciones sociales, culturales, económicas y políticas adversas afectan severamente las vidas de los usuarios, hacen que los efectos de una intervención profesional sean mucho menos predecibles que, por ejemplo, los efectos de un cierto fármaco o una intervención quirúrgica.

Las dificultades anteriores, si bien plantean la necesidad de ser consideradas, no opacan las potencialidades y la posibilidad de asumir este enfoque en la formación profesional. Sus adeptos insisten el hecho que la práctica basada en la evidencia permite mejorar la calidad en la prestación de servicios así como hacer un uso más racional de los recursos disponibles. Ella posibilitaría rebatir aquellas opiniones profesionales que sin contar con argumentos sólidos, se realizan a partir de prácticas comúnmente aceptadas: la autoridad, creencias imperantes, información anecdótica, o investigación de escasa calidad metodológica (Morago, 2004). Asimismo, la práctica basada en la evidencia en Trabajo Social respondería a un mandato ético (Gibbs \& Garambrill, 2002 en Furman, 2009), pues ella permitiría efectivamente incluir el estado del arte de los conocimientos, lo cual resulta clave para una intervención social compleja, innovadora y de calidad.

Ahora bien, Ainsworth y Hansen (2005), llaman la atención sobre el hecho que si se desea incluir en la formación de los trabajadores sociales la perspectiva de la práctica basada en la evidencia, se deben asumir, entre otros aspectos, ciertos compromisos en la estructura, contenidos curriculares y actuación de los docentes. Refiriéndose a este punto, los autores destacan:

- Que desde el inicio de la formación en Trabajo Social, se dé un énfasis mucho más marcado a la enseñanza de habilidades de pensamiento crítico.

- Que los académicos enfaticen y modelen como un valor básico del Trabajo Social, la construcción del conocimiento a través de la investigación.

- Que la enseñanza de la investigación, se convierta en un elemento básico en cada año de estudios conducente a una calificación en Trabajo Social. Los programas deben considerar que los estudiantes requieren demostrar habilidades tanto para el diseño de investigaciones, recopilación y análisis de datos, utilizando técnicas cualitativas y cuantitativas, como competencias para la práctica directa.

- Que los docentes se concentren en actividades de investigación, escritura y difusión del conocimiento a través de publicaciones especializadas. 


\section{Conclusiones}

Los hallazgos previos de la investigación "Infancia y riesgo social señalan que los Argumentos que utilizan los trabajadores sociales para tomar decisiones frente a situaciones de riesgo social que afectan al niño en su familia". Si bien estos hallazgos son preliminares y tienen una carácter exploratorio, pues surgen de una investigación pionera en Chile, dan la posibilidad de abrir una discusión acerca de la formación en Trabajo Social. Específicamente, ellos interpelan acerca de la necesidad de incorporar enfoques que permitan fortalecer los procesos de argumentación y de toma de decisiones en la intervención social. La estructura de análisis de la investigación permitió - por una parte- develar que en la argumentación profesional existiría un énfasis de los argumentos básicos por sobre los complementarios, denotando en los profesionales una falta de dominio en conocimientos de tipo predictivo validados por la disciplina, al momento de ponderar una situación y proponer una intervención social. Por otra parte, existiría también un déficit en el uso de la teoría y de la evidencia empírica, como soporte a la argumentación.

El déficit en el nivel complementario de argumentación se vincula a competencias basadas en la educación y el entrenamiento, que son claves para la actuación profesional. De ahí la necesidad de repensar la formación en Trabajo Social, introduciendo enfoques que permitan fortalecer el entrenamiento en habilidades de pensamiento crítico, así como en el uso de la teoría y de la evidencia empírica, como soporte a los juicios profesionales.

De lo anterior, se hace evidente la necesidad de formar a los estudiantes en marcos conceptuales que les permitan comprender y mejorar los procesos de toma de decisiones, así como el uso de metodologías que favorezcan el uso de la teoría y de la evidencia empírica en el ejercicio profesional. Al respecto, la teoría de la argumentación de Toulmin y el enfoque de la práctica basada en la evidencia, pueden constituirse en uno de los posibles aportes en este sentido. Sin embargo, ello requiere de innovaciones tanto en las prácticas pedagógicas como en los contenidos y estructuras curriculares, constituyéndose en un desafío a pensar por quienes ejercen docencia en Trabajo Social.

\section{Referencias}

AARON ROSEN, ENOLA K., PROCTOR, MARLYS M. STAUDT. (1999). Social Work Research. Washington. Tomo 23, No 1 ; pg. 4-14

AINSWORTH, F. \& HANSEN P. (2005). Evidence based social work prectice: a reachable goal?, en Bilson A., Evidence based practice in social work. London: Whiting \& Birch.

ALDAO, M. (2007). Teoría de la argumentación y democracia, en Revista Ambrosio I. Gioja Facultad de Derecho. Universidad de Buenos Aires año 1, $N^{\circ} 1$, pp. 8-16. http://www.derecho.uba.ar/revistagioja/articulos/ R0001A001_0002_investigacion.pdf

BRUNNER, J.J. (1993) ¿Contribuye la investigación social a la toma de decisiones? Conferencia pronunciada en el seminario "La Investigación Educacional Latinoamericana de cara al año 2000". Consejo Latinoamericano de Ciencias Sociales Comisión Educación y Sociedad, Punta de Tralca, 4-6 de junio de 1993.

BIAGINI, L. (2005). La evidencia y el proceso de toma de decisiones en Revista Médica de Chile, 2005; 133: 871-873.

COUTURIER, Y. \& CARRIER. S, (2003). Pratiques fondées sur les données probantes, en Travail social : un débat émergent, en Nouvelles Pratiques Sociales, Volume 16, numéro 2, p. 68-7, Éditeur : Université du Québec à Montréal.

BEDREGAL, P. \& CORNEJO C. (2005). El movimiento de la medicina basada en evidencia. Alcamnces conceptuales y teóricos, en Revista Médica de Chile; v. 133:977-982

FURMAN, R. (2009). Ethical Considerations of Evidence-Based Practice, en Social Work. Tomo 54, $\mathrm{N}^{\circ} 1$; Pg. 82,3

BENBENISHTY, P., OSMO R., GOLD N. (2003). Rationales Provided for Risk Assessments and for Recommended Interventions in Child Protection: A Comparison between Canadian and Israeli Professionals, in British Journal of Social Work; 33, pp, $137-155$

BIAGINI, A. (2005). La evidencia y el proceso de toma de decisiones, en Revista Médica de Chile v.133 No 8, ISSN 0034-9887.

FURMAN, R. (2009). Ethical Considerations of Evidence-Based, en Social Work. New York. Tomo 54, No 1 ; Pg. 82, 3 pgs.

GOLD, N. de la "The decisión to remove a child from the home in instantes of abuse or neglect. Professional decisión-making in Canada and Israel: a comparative study, Canada, McMaster University, 1998. 
LEITÃO, S. (2007). La dimensión epistémica de la argumentación. En Kronmüller, E. \& Cornejo, C. Ciencias de la Mente: Aproximaciones desde Latinoamérica. Santiago, Chile: J.C. Sáez Editor.

LUDI, M. (2005). Formación académica en Trabajo Social, en De Jong E. (2003), Formación académica en Trabajo Social. Una apuesta política para repensar la profesión, Buenos Aires: Espacio.

MARAFIOTI, R. (Compilador) (1998). Recorridos semiológicos. Signos, enunciación y argumentación. Buenos Aires, Eudeba.

MARAFIOTI, R. (2003). Los patrones de la argumentación. La argumentación en los clásicos y en el siglo XX. Buenos Aires: Biblos.

MIRANDA, P. (2006). Observaciones de teorías éticas en los procesos argumentativos de los trabajadores sociales, en Revista Trabajo Social, Santiago de Chile, Escuela de Trabajo Social, Pontificia Universidad Católica de Chile, pp. 83-97.

MIRANDA, C. (2003). El pensamiento crítico en docentes de Educación General Básica en Chile: un estudio de impacto. Estudios Pedagógicos (online). $\mathrm{N}^{\circ} \quad 29$, pp. 39-54.<http://www.scielo.cl/scielo. php?script=sci_arttext $\&$ pid $=$ S0718-07052003000100 003\&lng=es\&nrm=iso >. ISSN 0718-0705.

OSMO, R. Landau R., The need for explicit argumentation in ethical decision-making in social work in Social Work Education, Vol. 20 No $^{\circ}$, 2001.

OSMO, R. Benbennishty R, (2002). Children at risk: Rationales for risk assesments and interventions. Hebrew University, Jerusalem Israel.

OSMO, R. \& ROSEN A. (2002). Social workers' Strategies for treatment hypothesis testing in Social Work Research, Vol. 26, Nº1.

ROSEN, A., PROCTOR E., STAUDT M. (1999) Social work research and the quest for effective practice in Social Work Research, Washington, Tomo 23, № 1; pp. 4-11.

ROSEN, A., ENOLA K. PROCTOR, NANCY MORROW - HOWELL, MARLYS M. STAUDT. (1995). Rationales for Practice Decisions: Variations in Knowledge Use by Desicions Task and Social Work Services. Research on Social Work Practice. Vol. 5. N 4. Octubre 1995, $502-523$.

RODRÍGUEZ, L. (2004). El modelo argumentativo de Toulmin en la escritura de artículos de investigación educativa, en Revista Digital Universitaria. UNAM., Vol. 5, No1pp. 2-18. http://www.revista.unam.mx/ vol.5/num1/art2/ene_art2.pdf

SENAMÉ. (2005). Orientaciones Técnicas. CTD. Departamento de Protección de Derechos. Santiago, Ministerio de Justicia.
SENAME. (2005). Diagnóstico. Santiago de Chile, Ministerio de Justicia. www.sename.cl/interior/ diagnostico/f_diagnostico.html

SENAME. (2007). Protección Infancia y Adolescencia, Santiago de Chile, Ministerio de Justicia, www.sename.cl.

TOULMIN, S. (2007). Los usos del Argumento. Barcelona: Península. Primera edición en español.

TOULMIN, S., RIEKE R., JANIK A. (1984). An introduction to reasoning. (2 $2^{\text {da. }}$ Ed.) New York, Macmillan Publishing Co., Inc.

UNICEF. (2000). Maltrato infantil en Chile. UNICEF Responde, Santiago, Chile, www.unicef.cl/archivos_ documento/18/Cartilla\%20Maltrato\%20infantil.pdf

VALLE, M. \& CABRERA E. (2007). La argumentación como herramienta de enseñanza en clases de ciencias: una experiencia de laboratorio y en las aulas, en Boletín de Investigación Educacional. Vol. 22, N², pp.: 149-176. 